\title{
The Impact of MRI-TRUS Cognitively Targeted Biopsy on the Incidence of Pathologic Upgrading After Radical Prostatectomy
}

\author{
Ragheed Saoud ${ }^{\mathrm{a}}$, Albert El-Haj ${ }^{\mathrm{a}}$, Raja Khaulia, Muhammad Bulbula, b
}

\begin{abstract}
Background: The aim of the study was to evaluate the utility of multiparametric magnetic resonance imaging (mp-MRI)-transrectal ultrasound (TRUS) cognitively targeted biopsy in identifying the most significant cancerous lesion in the prostate to decrease the incidence of pathologic upgrading after radical prostatectomy.

Methods: We conducted a retrospective review of all radical prostatectomies at the American University of Beirut Medical Center between January 2016 and 2017. Pathology reports for both, TRUS biopsy and surgically resected specimens were analyzed and compared using SPSS.
\end{abstract}

Results: Among 66 patients who underwent radical prostatectomy, 44 patients underwent a standard random 12-core biopsy of the prostate, while 22 patients underwent 4 - 5 cognitively targeted biopsies. Biopsy Gleason scores were compared to surgically resected specimens. Of mp-MRI targeted biopsies, $86 \%$ were identical to the surgical specimen, while $14 \%$ were upgraded. Of the random biopsy, $55 \%$ patients upgraded after surgery, while $38 \%$ were concordant with the random biopsy result. Moreover, 13/24 patients who upgraded after random biopsy, did so from Gleason $6(3+3)$ to Gleason $7(3+4)$. The difference in pathological upgrading among both groups is statistically significant, and confirms the importance of MRI-TRUS cognitively targeted biopsy in identifying the highest risk lesion. This may have significant implications on the choice of treatment prior to embarking on surgical resection of prostate cancer.

Conclusion: MRI-TRUS targeted biopsy is more accurate than random biopsy in identifying the most significant cancerous lesion, resulting in a decreased incidence of pathologic upgrading after prostatectomy. This may have significant implications on the choice of treatment especially in low risk prostate cancer. Larger scale multicenter studies are required.

Keywords: Multiparametric; MRI; Targeted; Cognitive; Biopsy

Manuscript submitted January 16, 2018, accepted February 5, 2018

aAmerican University of Beirut Medical Center, Beirut, Lebanon

${ }^{\mathrm{b}}$ Corresponding Author: Muhammad Bulbul, American University of Beirut

Medical Center, Beirut, Lebanon. Email: mb30@aub.edu.1b

doi: https://doi.org/10.14740/wjnu285w

\section{Introduction}

Since the introduction of prostate-specific antigen (PSA) testing along with digital rectal examination, the proportion of patients diagnosed with early stage prostate cancer has exponentially risen. Prostate carcinoma is not a homogeneous disease. It can vary from being indolent and slow growing defined by D'Amico et al as low risk prostate cancer $[1,2]$ to being aggressive carcinoma that requires immediate therapy.

Due to this heterogeneity in prostate cancer biology, not all tumors require treatment (i.e. surgical or otherwise). If low risk prostate cancer is identified early (Gleason $\leq 6, \mathrm{PSA} \leq 10$, cT2a or less), it can be managed by active surveillance, hence avoiding surgery and more radical treatment strategies [3, 4].

Traditionally, the diagnosis of prostate cancer was achieved by random sampling of the gland via a sextant or random 12-core biopsy from its right and left lobes. However, multiple studies have shown that there is an increased risk of pathologic upgrading when preoperative biopsy specimens are compared to radical prostatectomy specimens [5].

The aim of this study was to investigate the impact of multiparametric magnetic resonance imaging (mp-MRI)/transrectal ultrasound (TRUS) guided cognitively targeted biopsy on the incidence of pathologic upgrading of prostate cancer after radical prostatectomy. To our knowledge, this is the first study of its kind to date.

\section{Materials and Methods}

After obtaining Institutional Review Board approval at the American University of Beirut Medical Center (AUBMC), a retrospective review of all patients who underwent radical prostatectomy for prostate adenocarcinoma was performed. Over a period extending from January 2016 until January 2017, a total of 66 patient charts were collected, 11 had undergone open radical prostatectomy (OPN) and 55 robotic-assisted radical prostatectomy (RARP). All 66 patients had biopsy proven clinically localized prostate adenocarcinoma at our institution prior to undergoing surgical extirpation. Patients who had undergone TRUS biopsy outside AUBMC and those who were selected for active surveillance or were subject to androgen deprivation/radiation therapy were excluded. Not all TRUS biopsy specimens were read by the same pathologist, and in the 


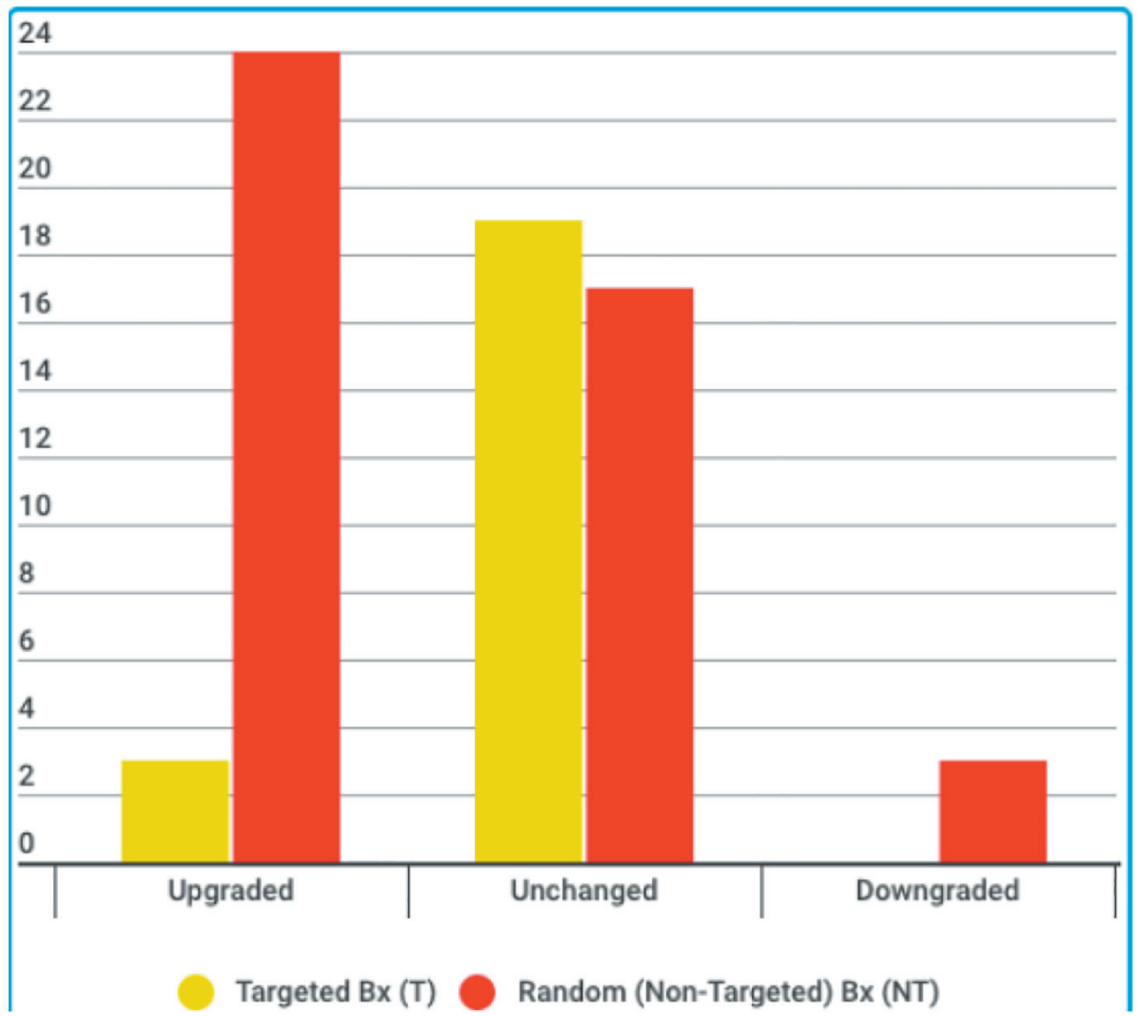

Figure 1. Comparison of pathological grade after prostatectomy in targeted versus random biopsy patients.

majority of cases, different pathologists read the biopsy and its respective surgically resected specimen. Patients with missing pathological data were also excluded. Moreover, all those who underwent mp-MRI and proceeded to MRI/TRUS cognitive biopsy were identified and their reports were collected and reviewed. The data gathered from pathology reports were also reviewed and analyzed using the latest SPSS software (version 22.0.0.0). Pearson Chi-square tests were performed and P-values were calculated.

\section{Results}

Sixty-six patients underwent radical prostatectomy for clinically localized prostate cancer at AUBMC over a period extending from January to the end of December 2016. Twentytwo patients had undergone an mp-MRI and consequently, a cognitively targeted TRUS biopsy, where 4 - 5 cores were sampled from the most suspicious highest risk lesion identified on MRI from 1 lobe, and 2 - 3 random cores from the contralateral lobe. The remaining 44 patients underwent a conventional 12-core random biopsy with three cores sampled from each of the right and left, transitional and peripheral zones, respectively. Among those who underwent targeted biopsy (T), $3 / 22(13.6 \%)$ patients witnessed pathological Gleason score upgrading after radical prostatectomy, while $24 / 44$ (54.5\%) of those in the conventional biopsy (B) group were upgraded; the difference in pathological upgrading after surgery is statistically significant with a P-value $<0.001$ (Fig. 1). To note also, that $38 \%(17 / 44)$ of the random biopsy group had identical Gleason scores after surgery, while $86 \%(19 / 22)$ of those in the targeted biopsy group remained unchanged.

In the subgroup of patients who underwent pathological upgrading after random biopsy, Gleason 6 to Gleason 7 $(3+4)$ upgrading was noted in 54\% (13/24) (Fig. 2). According to the D'Amico risk stratification criteria, this is a shift from low to intermediate risk prostate cancer (7.1). Moreover, $33 \%(8 / 24)$ upgraded from Gleason $7(3+4)$ to Gleason $7(4+3)$ and Gleason 8 , while the remaining patients $(3 / 24)$ upgraded from Gleason $8(4+4)$ to Gleason 9. On the other hand, none of the patients diagnosed with Gleason $6(3+3)$ prostate adenocarcinoma on targeted biopsy eventually upgraded. Such unexpected differences in pathologic outcome after surgery between the two groups may have major implications on the choice of treatment preoperatively.

\section{Discussion}

The diagnosis and treatment of prostate cancer has significantly evolved over the past decade. Traditionally, a suspicious digital rectal examination along with an elevated serum PSA level indicated a need for prostate biopsy. However, random 12-core biopsies under TRUS guidance do not accurately tar- 


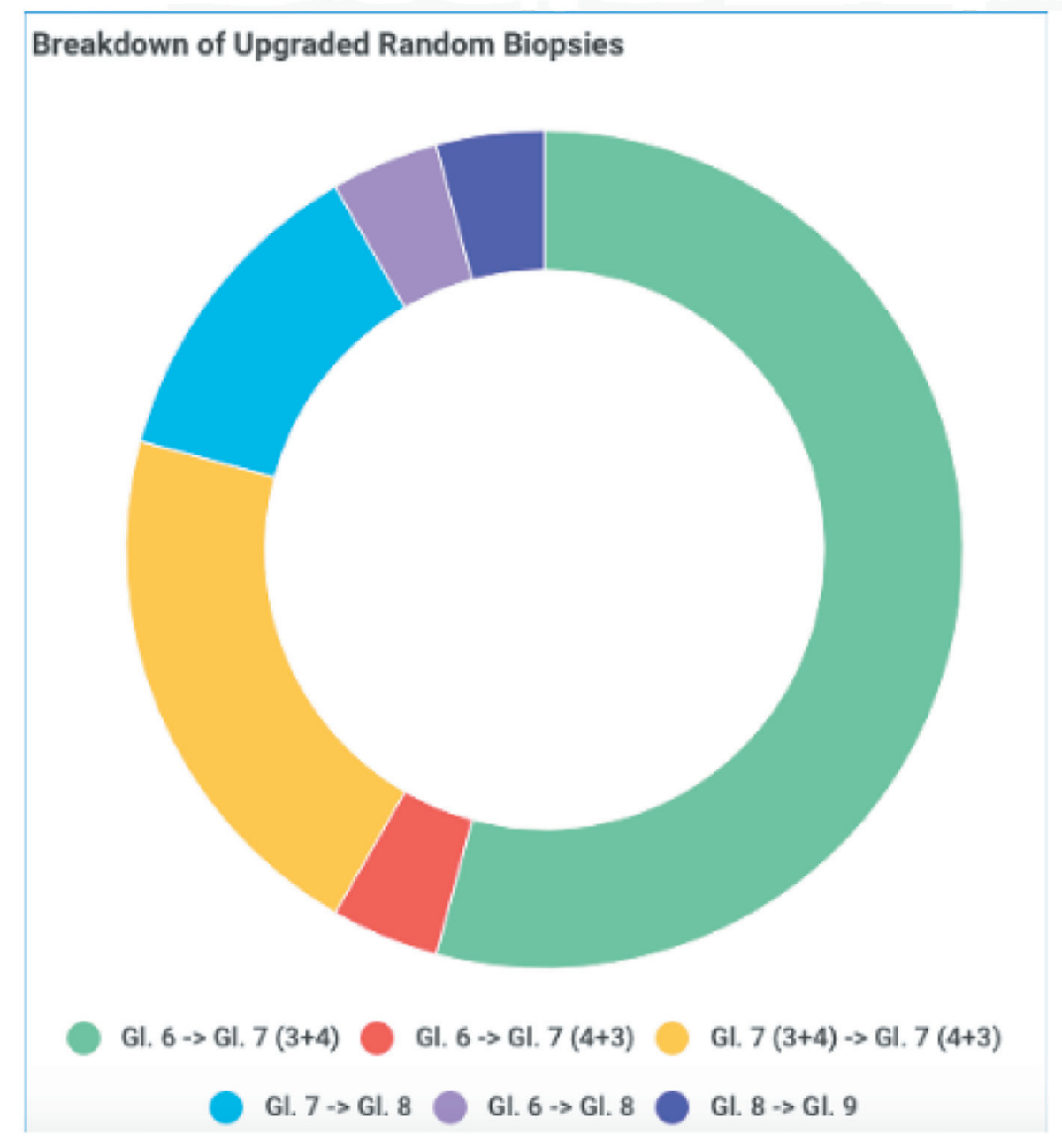

Figure 2. Breakdown of upgraded random biopsies after radical prostatectomy.

get a specific area of the prostate but rather sample the gland as a whole. This however does not come without risks. In addition to the increased risk of bacteremia due to the large number of cores required to sample the gland, random sampling has also proven to be a major risk factor for pathologic Gleason upgrading after radical prostatectomy [6].

In most contemporary series, the risk of upgrading in patients with low risk disease who undergo surgery is in the range of 40-55\% [7]. Pathologic upgrading is concerning especially in patients who are diagnosed with low risk prostate cancer on biopsy. Patients with a Gleason score $\leq 6$, clinical stage $\leq$ $\mathrm{T} 2 \mathrm{a}$, and serum PSA $\leq 10$ are stratified as low risk according to the D'Amico and PRIAS risk stratification criteria. Due to the indolent slow growing nature of prostate cancer, these patients are usually candidates for active surveillance based on their diagnostic biopsy. However with the increased rate of pathologic upgrading that is apparent in radical prostatectomy specimens, it is imperative that the most suspicious lesion is diagnosed preoperatively to correctly reflect Gleason score and thus clinical stage. This will enable the physician to decide on the most appropriate therapeutic option prior to embarking on surgery.
The introduction of mp-MRI with diffusion-weighted imaging allows urologists to modify their algorithm for diagnosing prostate cancer. In biopsy naive men suspicious of harboring prostate cancer, mp-MRI has $96 \%$ sensitivity in detecting clinically significant disease, with a negative predictive value of $92 \%$ [8]. This allows urologists to adopt mp-MRI as an integral part of their diagnostic armamentarium prior to proceeding directly to random TRUS biopsy. The value of mp-MRI is in the detection of clinically significant disease in the prostate and scoring it based on the PI-RADS classification; lesions given the score of $1-2$ are usually benign, while those with a score of 4 - 5 are most probably malignant and highly suspicious of harboring prostate carcinoma.

The value of mp-MRI is not only in the radiological diagnosis and risk stratification of prostate cancer. It allows the urologist to target radiologically significant disease during TRUS biopsy, a feature that is not possible using conventional ultrasound guidance alone [9, 10]. From the urologist's perspective, radiologically significant disease is that highest risk lesion in the prostate that determines the Gleason grade and stage, and hence aids in deciding on the appropriate course of 
treatment. Our aim was to assess the utility of MRI in minimizing the discrepancy between biopsy findings and surgically resected specimens by more accurately detecting clinically significant prostate cancer using MRI/TRUS cognitive guidance. This should have implications, not only on the diagnosis and stratification of patients into their correct risk category, but also on the adequate choice of treatment. Our data show that among the patients in the random biopsy group, 14/44 (32\%) upgraded from Gleason 6 to Gleason 7, which in turn upstages their disease from low to intermediate risk. Such patients indeed harbor disease that is not eligible for active surveillance. This comes to show that urologists may not be able to depend on random 12-core biopsies for the accurate staging of prostate cancer prior to performing radical prostatectomy. Moreover, the high sensitivity of mp-MRI in correctly detecting clinically significant disease is advantageous as it permits correct staging prior to surgery. Our data show that none of the patients with Gleason score 6 after mp-MRI-TRUS cognitive biopsy was eventually upgraded. Those patients are considered low risk and underwent active surveillance rather than proceeding to surgery. We strongly believe that in such situations where histological grade of the biopsy determines the choice of treatment, mp-MRI fused with TRUS is essential in our diagnostic armamentarium. Moreover, in the subgroup of patients who underwent targeted biopsy $(n=22)$, the protocol dictates that 2-3 random cores are sampled from the contralateral lobe. None of the patients had a random biopsy core with a Gleason score higher than the target biopsy, which further portrays the accuracy of mp-MRI in detecting the highest risk lesion in the prostate and thus targeting it with TRUS cognitive guidance.

To our knowledge, this study is the first of its kind to address the influence of MRI-TRUS cognitive fusion to decrease the incidence of pathologic upgrading. The results described above are encouraging as they advocate the use of MRI and highlight its importance in the accurate diagnosis of prostate cancer, especially in low risk localized disease. In addition, virtually all previously reported data on upgrading were based on subjects who received sextant biopsy, which is not reflective of contemporary practice (Hong). The aim was to decrease the number of unnecessary cores from areas of the gland that are not highly suspicious for tumor, while maintaining an excellent sensitivity for diagnosis of clinically significant prostate cancer.

We note several caveats to our study. Apart from its retrospective nature and its short duration, this study is single institutional. The subjects reviewed are mostly Lebanese and do not reflect the variable racial disparities of prostate cancer biology. In addition, even though all subjects underwent radical prostatectomy, the surgical technique utilized was not uniform, which may affect the final specimen retrieved and its pathologic outcome. The TRUS biopsy and radical prostatectomy specimens were not examined and graded by the same pathologist, which may also be a source of bias in our results. We also acknowledge that our data may not be reflective of low risk disease as a whole, because many of our patients who underwent surgery are candidates for active surveillance.

The introduction of mp-MRI and its high sensitivity in tumor detection is considered an upgrade in the diagnostic armamentarium of prostate cancer. Due to the indolent nature of this disease and the availability of active surveillance as an option for its treatment, the preoperative grade and stage must be accurately evaluated prior to therapeutic decision-making, especially in patients harboring low risk prostate cancer. We believe that MRI-TRUS cognitively targeted biopsy can play a significant role in the accurate diagnosis of prostate cancer by decreasing the risk of pathologic upgrading after radical prostatectomy. Larger randomized prospective studies are required to further validate our hypothesis and generalize our results.

\section{Conflict of Interest}

None of the authors has any interests to disclose.

\section{References}

1. Hardie C, Parker C, Norman A, Eeles R, Horwich A, Huddart R, Dearnaley D. Early outcomes of active surveillance for localized prostate cancer. BJU Int. 2005;95(7):956-960.

2. Soloway MS, Soloway CT, Eldefrawy A, Acosta K, Kava B, Manoharan M. Careful selection and close monitoring of low-risk prostate cancer patients on active surveillance minimizes the need for treatment. Eur Urol. 2010;58(6):831-835.

3. Klotz L, Zhang L, Lam A, Nam R, Mamedov A, Loblaw A. Clinical results of long-term follow-up of a large, active surveillance cohort with localized prostate cancer. J Clin Oncol. 2010;28(1):126-131.

4. van den Bergh RC, Roemeling S, Roobol MJ, Aus G, Hugosson J, Rannikko AS, Tammela TL, et al. Outcomes of men with screen-detected prostate cancer eligible for active surveillance who were managed expectantly. Eur Urol. 2009;55(1):1-8.

5. Freedland SJ, Kane CJ, Amling CL, Aronson WJ, Terris MK, Presti JC, Jr., SEARCH Database Study Group. Upgrading and downgrading of prostate needle biopsy specimens: risk factors and clinical implications. Urology. 2007;69(3):495-499.

6. Busch J, Magheli A, Leva N, Ferrari M, Kramer J, Klopf $\mathrm{C}$, Kempkensteffen $\mathrm{C}$, et al. Higher rates of upgrading and upstaging in older patients undergoing radical prostatectomy and qualifying for active surveillance. BJU Int. 2014;114(4):517-521.

7. Hong SK, Han BK, Lee ST, Kim SS, Min KE, Jeong SJ, Jeong $\mathrm{H}$, et al. Prediction of Gleason score upgrading in low-risk prostate cancers diagnosed via multi $(>$ or $=12)$ core prostate biopsy. World J Urol. 2009;27(2):271-276.

8. Ahmed HU, El-Shater Bosaily A, Brown LC, Gabe R, Kaplan R, Parmar MK, Collaco-Moraes Y, et al. Diagnostic accuracy of multi-parametric MRI and TRUS biopsy in prostate cancer (PROMIS): a paired validating confirmatory study. Lancet. 2017;389(10071):815-822.

9. Hambrock T, Hoeks C, Hulsbergen-van de Kaa C, Scheenen T, Futterer J, Bouwense S, van Oort I, et al. Prospective assessment of prostate cancer aggressiveness using 3-T diffusion-weighted magnetic resonance 
imaging-guided biopsies versus a systematic 10-core transrectal ultrasound prostate biopsy cohort. Eur Urol. 2012;61(1):177-184.
10. Boesen L, Thomsen HS. [Magnetic resonance imaging in management of prostate cancer]. Ugeskr Laeger. 2013;175(23):1630-1633. 